\title{
O FENÔMENO DA JUDICIALIZAÇÁO E O ACESSO A MEDICAMENTOS DE ALTO CUSTO NO BRASIL: UMA REVISÃO SISTEMÁTICA DA LITERATURA
}

\author{
THE PHENOMENON OF JUDICIALIZATION AND ACCESS TO HIGH COST MEDICATIONS \\ IN BRAZIL: A SYSTEMATIC REVIEW OF THE LITERATURE
}

Silvia Maria Aparecida Vitorino

Mestre em Tecnologias da Informação e Comunicação Instituição de Ensino pela Universidade Federal de Uberlândia - UFU

Administradora - DPU Uberlândia

silviavitorino2006@yahoo.com.br

\section{RESUMO}

O fenômeno da judicialização da saúde no Brasil é recente, porém tomou grande vulto nas duas últimas décadas, como consequência das demandas judiciais que obrigam o Estado a prover de bens e serviços de saúde, inclusive o fornecimento de medicamentos de alto custo para tratar doenças raras. Objetiva-se nesse estudo traçar o perfil das produções acadêmicas sobre a judicialização do acesso à medicamentos de alto custo no Brasil, analisando estudos publicados nos últimos quatorze anos em base de dados primárias como Google Scholar,Acadêmico, que segmentaram em trabalhos de fontes secundárias como Scielo, Medline, Revistas de Saúde Pública, de Direito Sanitário, Fiocruz, Brazilian Political Science, Escola de Saúde Pública, além de trabalhos vinculados a Universidades Públicas Federais, (CAPES) - Coordenação de Aperfeiçoamento de Pessoal de Nível Superior, que juntos compuseram a amostra. Para traçar esse perfil, realizou uma revisão sistemática com teses de doutorado, dissertaçóes de mestrado, artigos científicos e capítulos de livros. A amostra perfaz 69 estudos com uma produçáo progressiva desde 2005, em todas as regióes do país, com destaque para a região sudeste, ganhando amplo enfoque na área da saúde. Os resultados apontaram que essa demanda acarreta interferências nas políticas públicas comprometendo o orçamento da saúde, porém é uma estratégia para o enfrentamento do problema. $\mathrm{O}$ perfil das produçóes acadêmicas versa sobre as características dos requerentes, o comportamento similar nas decisóes judiciais e, ainda, sobre o percentual dos medicamentos requeridos que apresentam alternativa terapêutica - (79\%), além das dificuldades apresentadas pelos órgãos públicos para o cumprimento dessas decisões judiciais. 
Palavras-chave: Judicialização. Medicamentos. Sistema único de Saúde. Ações Judiciais.

\begin{abstract}
The phenomenon of judicialization of health in Brazil is recent, but took a great figure in recent years, more specifically in the past two decades, as a result of lawsuits to compel the State to provide health facilities, goods and services, including the supply of high cost medications for the treatment of rare diseases. The objective of the present study was to trace the profile of academic productions on the judicialization of access to medicines for high cost in Brazil, analyzing the studies published in the last fourteen years on the basis primary database such as Google Scholar, which segmentaram in works of secondary sources such as Scielo, Lilacs, Medline, magazines of Public Health, Fiocruz, Journal of Health Law, Brazilian Political Science, School of Public Health, as well as jobs linked to Federal Public Universities, and the (CAPES) - [Higher Education Personnel Improvement Coordenation] that comprised the sample analyzed. To trace this profile, we conducted a systematic review with theses doctoral dissertations, scientific articles and chapters of books. The sample makes 69 studies with a progressive production since 2005 , in all regions of the country, with emphasis on the southeastern region, gaining wide results showed that this demand causes interference in public policies affecting the health budget, but it is also a strategy for dealing with the problem. The profile of the academic productions focus on the focus in the area of health. The characteristics of applicants, the similar behavior in judicial decisions, and also about the percentage of the required medicines which have a therapeutic alternative - (79\%), in addition to the difficulties presented by public bodies for compliance with these judgments.
\end{abstract}

Keywords: judicialization. Medicines. System Unique of Health. lawsuits.

Data de submissão: 30/05/2019

Data de aceitaçáo: 17/01/2020

\title{
SUMÁRIO
}

INTRODUÇÃO 1. POLÍTICA DE MEDICAMENTOS E O DEVER LEGAL DO ESTADO NO FORNECIMENTO DE MEDICAMENTOS DO SUS 2. ASPECTOS METODOLÓGICOS 3. RESULTADOS E DISCUSSÃO. CONCLUSÃO. 


\section{INTRODUÇÁO}

Embora o direito à saúde esteja contemplado no texto constitucional de 1988, mais especialmente no artigo $196,{ }^{1}$ preceitua:

O art.196 da Constituiçāo Federal aponta que a saúde é direito de todos e dever do Estado, garantido mediante políticas sociais e econômicas que visem à redução do risco de doença e de outros agravos e ao acesso universal e igualitário às açôes e serviços para sua promoção, proteção e recuperação.

Nesse prisma, carências de natureza gerencial, administrativa e orçamentária, entre outras, restringem a capacidade operativa dos órgãos e entidades responsáveis pela sua efetivação desencadeando um fenômeno denominado judicialização do direito à saúde. Este fenômeno é observado quando os indivíduos adotam a iniciativa de recorrer ao Poder Judiciário para garantirem o direito de acesso aos meios e recursos necessários a melhoria de suas condiçôes clínicas. Como o atendimento a saúde deve ser integral, contemplando todas as necessidades do indivíduo, o fenômeno também é observado quando o paciente recorre à justiça para garantir o direito de acesso a medicamentos que deveriam ser fornecidos gratuitamente pelo poder público. ${ }^{2}$

A norma constitucional prevê a saúde como direito de todos e dever do Estado, garantido mediante políticas sociais e econômicas. Ademais, o tipo constitucional impōe o acesso universal e igualitário às açôes e serviços para a promoção, proteção e recuperação da saúde.

Luiz Alberto David de Araújo e Vidal Serrano Nunes Júnior, ao tratarem do tema, doutrinam:

$\mathrm{O}$ art. $6^{\circ}$ da Constituiçáo Federal indicou a saúde como um direito social incluindo-o, portanto, no rol dos chamados Direitos funda-mentais. Na verdade, o direito à saúde constitui um desdobramento do próprio direito à vida, logo, por evidente, não poderia deixar de ser considerado como um direito fundamental do indivíduo. Nesse sentido, o art.196 prescreve que a saúde é direito de todos e um dever do Estado, criando, por assim dizer, um direito subjetivo público de atençáo à saúde, cuja tutela tanto pode dar-se pela via coletiva como pela individual. ${ }^{3}$

No Brasil, segundo Wannmacher (2010), Cooper et al (2009), Bonfim (2008), o acesso a medicamento é parte do direito à saúde, por se tratar de um bem importante para o cuida-

BRASIL. Constituiçáo Federal. Planalto

2 JUNIOR, P.B.R. A Judicializaçáo do Acesso a Medicamentos - A Perspectiva da Secretaria Municipal de Saúde do Rio de Janeiro, 2008. p.51.

3 ARAUJO, l. A. D.; NUNES JÚNIOR, V. S. Curso de Direito Constitucional, 2005, p. 584. 
do, por seu potencial para prevenir e curar doenças ou até salvar vidas. ${ }^{4,5,6}$ No mesmo eixo, Oliveira (2007), aponta que a garantia do acesso, contudo, é uma questão complexa que depende do contexto econômico, político e social. A situação mundial revela uma grave iniquidade no acesso: apenas $10 \%$ da população mundial consomem $90 \%$ da produção de medicamentos.

De modo geral, Pessoa (2007) revela que a melhoria do acesso está associada à das condiçóes socioeconômicas, especialmente da distribuição de renda, mas também à capacidade de financiamento dos sistemas de saúde, ao uso racional dos medicamentos e à eficiência na gestão dos recursos. Ademais, características próprias da área farmacêutica representam desafios à garantia do acesso: a complexidade da seleção de quais produtos ofertarem, a opacidade dos mecanismos de definição de preços e as dificuldades de desenvolvimento de açôes efetivas para promover o uso racional. ${ }^{8}$

A Política de Assistência Farmacêutica do Sistema Único de Saúde - (SUS) define as diretrizes de gestão para assegurar o fornecimento e o uso racional de medicamentos. Atualmente, encontra-se organizada em três componentes: básico - medicamentos para o tratamento de doenças mais prevalentes; estratégico - medicamentos para o tratamento de endemias e Aids; e especializado - medicamentos cuja utilização depende da observaçáo de Protocolos Clínicos e Diretrizes Terapêuticas?. Apesar de tecnicamente consistente, Figueiredo et al (2010), aponta que a Política de Assistência Farmacêutica não tem conseguido atender às necessidades de acesso a medicamentos, por uma série de fatores, entre os quais se podem citar: o desconhecimento ou a náo adesão dos prescritores às listas de medicamentos disponíveis, as falhas na oferta tempestiva e a pressão do setor produtivo em prol do uso de fármacos não contemplados nas listas. ${ }^{10}$

As dificuldades de acesso explicam, em parte, o recurso dos usuários do SUS ao Poder Judiciário. A intervenção do Poder Judiciário, mediante determinações à Administração Pública, almeja, primordialmente, a concretizaçáo dos direitos previstos na Constituição. Essa intervenção pode ser chamada de judicialização, quando, por sua magnitude ou abrangência, implica a transferência para o Judiciário de decisões que caberiam aos Poderes Executivo e Legislativo. ${ }^{11}$ Em Vianna (2002), a judicialização é a resposta dos cidadãos, ressalta que quando o Estado não cumpri com suas obrigaçôes, sendo o Judiciário

4 WANNMACHER,L. Importância dos Medicamentos Essenciais em Prescriçáo e Gestáo Racionais, 2010. p.32.

5 COOPER, H. M.; HEDGES, L. V. The Handbook of research synthesis and meta-analysis. New York: The Russel Sage Foundation, 2009.

6 BONFIM,R.L.D. Agenda Única de Saúde: a busca do acesso universal e a garantia do direito à saúde. 2008, p.172-185.

7 OLIVEIRA,M.A.;BERMUDEZ,J.A.Z.;OSORIO-DE-CASTRO,C.G.S. Assistência Farmacêutica e acesso a medicamentos, 2007, p.132.

8 PESSOA,N.T. Perfil das solicitaçóes administrativas e judiciais de medicamentos impetradas contra a secretaria de saúde do estado do Ceará, 2007, p.168-185.

9 BRASIL. Assistência farmacêutica no SUS, 2011. p.186.

10 FIGUEIREDO, T.A.; PEPE, V.L.E.; OSORIO-DE-CASTRO, C.G.S. Um enfoque sanitário sobre a demanda judicial de medicamentos,2010, p.101-118.

11 MACHADO,F.R.S. A judicializaçáo da saúde no Brasil: cidadanias e assimetrias. 2010, p.187. 
a última opção para reivindicar seus direitos. ${ }^{12}$

Destarte, o fenômeno da judicialização da saúde no Brasil é recente, porém tomou grande vulto nas duas últimas décadas, como consequência das demandas judiciais que obrigam o Estado a prover de bens e serviços de saúde, inclusive o fornecimento de medicamentos de alto custo para tratar doenças raras. Objetiva-se nesse estudo traçar o perfil das produçóes acadêmicas, por meio de seus objetivos e resultados apresentados sobre a judicialização do acesso à medicamentos de alto custo no Brasil, analisando estudos publicados nos últimos dez anos em base de dados primárias como Google Acadêmico, que segmentaram em trabalhos de fontes secundárias como Scielo, Lilacs, Medline, Revistas de Saúde Pública, de Direito Sanitário, Fiocruz, Brazilian Political Science, Escola de Saúde Pública, além de trabalhos vinculados a Universidades Públicas Federais, (CAPES) - Coordenação de Aperfeiçoamento de Pessoal de Nível Superior, que juntos compuseram a amostra. Para traçar esse perfil, realizou uma revisão sistemática com teses de doutorado, dissertaçóes de mestrado, artigos científicos e capítulos de livros.

As açóes judiciais que pleiteiam o acesso a medicamentos têm tomado grande vulto, causando impactos significativos na estruturação, no financiamento e na organizaçáo do sistema de saúde, Sant’ana (2011). Iniciaram-se com açóes que exigiam o fornecimento de medicamentos para o tratamento da Aids, estratégia bem-sucedida, que se estendeu, a seguir, para as mais variadas doenças. ${ }^{13}$

O tema envolve questóes legais, éticas, políticas, econômicas e sociais, no que se refere ao dever do Estado, à escassez dos recursos e ao conflito entre direitos individuais e direitos coletivos. ${ }^{14}$

A partir desse contexto, o objeto de pesquisa é a judicialização de medicamentos de alto custo no Brasil. As perguntas de pesquisa consistem em responder: qual o perfil das produçôes acadêmicas sobre a judicialização do acesso a medicamentos de alto custo no Brasil?

A partir disso foram analisados e apresentados os resultados da pesquisa realizada entre os meses de março a dezembro de 2018, tendo como suporte as bases de dados expostas na metodologia.

\section{POLÍTICA DE MEDICAMENTOS E O DEVER LEGAL DO ESTADO NO FORNECIMENTO DE MEDICAMENTOS DO SUS.}

Sendo a saúde dever do estado a sua garantia, os medicamentos para tratamento de do-

12 VIANNA,L.W. A democracia e os três poderes no Brasil, 2002, p. 17-42.

13 SANT'ANA,J.M.B.et al. Essencialidade e assistência farmacêutica: consideraçôes sobre o acesso a medicamentos mediante ações judiciais no Brasil, 2011, p.158.

14 CATANHEIDE;I.D.LISBOA,E.S.SOUZA,L.E.P.F. Característica da Judicializaçáo do acesso a medicamentos no Brasil: Uma revisão sistemática, 2018, p.67-85. 
enças, ou prevenção das mesmas, vêm embutidos na concessão desse direito, de forma integral e igualitária. ${ }^{15} \mathrm{O}$ Art. $196 \mathrm{da}$ CF, diz que a saúde é direito de todos e dever do Estado, garantido mediante políticas sociais e econômicas que visem à redução do risco de doença e de outros agravos e ao acesso universal e igualitário às açóes e serviços para sua promoção, proteção e recuperação.

Nesse enfoque, é dever do estado garantir a saúde ao cidadão, assim sendo, é inconcebível a recusa do fornecimento de medicamentos a pacientes que deles necessitem, principalmente aqueles em estado grave e, sem condiçóes financeiras de custear tais despesas para o acesso a esses remédios, cuja função destes é retomar seu estado de saúde pleno.

Havendo, assim, entre os entes federativos, o Estado, a União e o Município, uma obrigatoriedade de fornecimento desses medicamentos entre sua população.

No escopo de cumprir o seu dever legal, o sistema único de saúde (SUS) oferece vários medicamentos gratuitos para a população, desde que conste na relação de uma lista de medicamentos, denominada de Relação Nacional de Medicamentos Essenciais - RENAME, credenciada pelo Estado e implantada nas secretarias e postos de saúde. A publicação dessa lista é feita pelo Ministério da Saúde, com os medicamentos para combater as doenças mais comuns que atingem a sociedade brasileira. Tem-se o objetivo de assegurar o acesso da população à medicamentos aos quais não teriam condiçôes de pagá-los, ou ainda fornecer de modo gratuito independente da situação financeira.

Desta forma, a pessoa deve comprovar a necessidade do medicamento, através de receita médica atualizada, por médico credenciado pelo sistema único de saúde, e ainda, através de exames de diagnósticos. ${ }^{16}$

Salazar et al (2008), Boing et al (2010), Chieff et al (2009), apontam ter ainda de ser lembrado que o médico, por meio de seu relatório, fundamentadamente, tem que comprovar a eficácia e segurança do medicamento determinado para aquele tratamento, além de registrado na Agência Nacional de Vigilância Sanitária - ANVISA. ${ }^{17,18,19}$

\section{ASPECTOS METODOLÓGICOS}

Para atingir os objetivos do estudo e ainda responder ao problema de pesquisa proposto,

15 BRANDÃO,L.S. A judicializaçáo da saúde quanto ao fornecimento de medicamentos, 2015, p.5-8.

16 Idem

17 SALAZAR, et al. A judicializaçáo dos anti-retrovirais no Brasil. In: BRASIL. Ministério da Saúde. Secretaria de Vigilância em Saúde,2008, p.43-52.

18 BOING, A.C.;BLOEMER,N.S.;ROESLER, C. O. Dilema do poder judiciário: Acesso e direito aos medicamentos (the dilemma of the judiciary: Law and acess to medicines), 2010, p.67-85.

19 CHIEFFI,A.L.;BARATA,R.B. Judicializaçáo da política pública de assistência farmacêutica e equidade, 2009, p.142-156. 
será realizada uma revisão sistemática de todos os trabalhos publicados sobre o processo de judicializaçáo do acesso a medicamentos de alto custo no Brasil, adotou-se a seguinte estrutura: (a) identificação da base de dados-fontes para consulta; (b) critérios de inclusão-exclusão pela leitura do título, leitura do resumo e finaliza com a leitura do texto completo; (c) objetivos da pesquisa; (d) resultado da pesquisa; (e) consideraçóes finais.

A pergunta que norteou o desenvolvimento deste estudo foi composta da seguinte forma: qual o perfil das produçóes acadêmicas sobre a judicialização do acesso a medicamentos de alto custo no Brasil? O estudo foi distribuído nos últimos quatorze anos, a instituição de filiação do principal autor, a localização da instituição por região do Brasil, o tipo de publicação, os descritores utilizados na pesquisa, os objetivos da pesquisa como primeira abordagem de escolha e os resultados das pesquisas como segunda abordagem de escolha, uma abordagem não exclui a outra, cabe ressaltar que ambas são complementares e não excludentes, ou seja, em algumas pesquisas não existem a segunda abordagem, apenas a primeira, pois a pesquisa pode ainda encontrar-se em desenvolvimento no momento da busca.

A base de dados foi composta por fontes primárias e retiradas do Google Scholar e Google Acadêmico, que segmentaram em trabalhos de fontes secundárias como Revista de Saúde Pública, Fiocruz, Revista de Direito Sanitário, Scielo, Brazilian Political Science Review, Cadernos de Saúde Pública, Livro de Direito Sanitário: Saúde e Direito, Ministério da Saúde, Physis, Universidade Estadual do Rio de Janeiro, Universidade Federal Fluminense, Universidade de Brasília, Revista Âmbito Jurídico, Revista Amicus Curiae, Biblioteca Digital da FGV, Constituição Federal, Revista Ciência e Saúde Coletiva, Conasems Ministério da Saúde, Revista Direito e Justiça, Faculdade Estácio de Sá, Revista Femina, Fundação Getúlio Vargas, Revista Health \& Social Change, Revista Health and Human Rights, Instituto de Saúde, Curso de Direito Constitucional, Medline, PUC - Salvador, Revista Brasileira de Políticas Públicas Uniceub, Revista Brasileira de Cirurgias Cardiovasculares, Revista de Medicina de Minas Gerais, Revista Panamericana de Salud Publica, Revista Associação Médica Brasileira, Revista AJURIS, Revista BVS - Brasileira de Vigilância em Saúde, Revista de Direito Sanitário, Revista DPU - Defensoria Pública da União, The Russel Sage Foundation, Universidade Federal da Bahia, Universidade Federal do Ceará, Universidade Federal de Góias, Universidade Federal de Minas Gerais, Universidade Federal de Pernambuco, Universidade Federal do Rio Grande do Sul,Universidade Federal de Santa Catarina, Univale, Universidade de Marília, Universidade de São Paulo.

O critério de inclusão-exclusão foi composto por títulos com os seguintes descritores: medicamento; judicialização; ações judiciais; decisóes judiciais; health; medicine; judicial, legal, de forma combinada e descrita abaixo:

1. Google Scholar (judicialização AND medicamento AND sistema único de saúde, judici\$ AND medicament\$), judici\$ AND decis\$ judici\$,

2. Google Acadêmico (judicialização AND medicamento AND sistema único de saúde, judici\$ AND medicament\$), judici\$ AND decis\$ judici\$,

3. Medline: ((“medicine”[MeSH Tems] OR "medicine”[All Fields]) AND judicial [All Fields]

4. Portal CAPES: judialização, medicamentos, medicamentos ações judiciais, decisóes 
judiciais, demandas judiciais;

5.Scielo: (judicia \$ND medicament\$, judic\$ AND saúde\$, direito AND medicamento AND açôes judiciais).

A busca foi realizada entre os meses de Março a dezembro de 2018 e com as estratégias utilizadas nas cinco bases de dados acima foi possível localizar $(\mathrm{n}=2382)$ resultados.

Destes, $(\mathrm{n}=1852)$ foram excluídos pela leitura do título, quando tratavam do tema da judicializaçáo da saúde, porém não voltados para o acesso a medicamentos, ou de estudos em duplicidade, ou ainda por não estarem disponíveis na íntegra.

Restaram ( $\mathrm{n}=530$ ) estudos que passaram por um segundo nível de análise, baseado na leitura do resumo e, nessa parte da pesquisa, foram retirados aqueles que tratavam da judicialização da saúde, porém não abordavam prioritariamente o acesso a medicamentos de alto custo no Brasil. Nessa etapa foram excluídos $(n=460)$ trabalhos, restando $(n=69)$ estudos, que foram selecionados por cumprirem todas as etapas. Ao final da leitura do texto na íntegra foram identificados 5 teses de doutorado, 11 dissertaçóes de mestrado, 51 artigos e 1 legislação, 1 livro.

A etapa final que compôs a análise, foi realizada a extração dos dados com a utilização das etapas elaboradas para o princípio da inclusão-exclusão no intuito de traçar o perfil das produções científicas que abordam a judicialização do acesso a medicamentos de alto custo no Brasil. Os dados coletados foram submetidos à estatística descritiva, utilizando percentagem, para amostras independentes, analisados por categoria, de acordo com os critérios pré-estabelecidos.

Esses dados podem ser vislumbrados e estratificados conforme figura 01 abaixo:

Figura 01: Tabela de Publicaçóes Coletadas

\begin{tabular}{|c|c|c|c|c|c|}
\hline AUTORES & BASE DE DADOS & FONTE & ANO & PUBLICAÇÓES & PERCENTAGEM \\
\hline LOPES, et al & Rev Saude Publica & Artigo & 2010 & \multirow{8}{*}{8} & \multirow{8}{*}{$11,58 \%$} \\
\hline MACEDO, et al & Rev Saude Publica & Artigo & 2011 & & \\
\hline MACHADO, M. & Rev Saude Publica & Artigo & 2011 & & \\
\hline SANT'ANA, J.M.B & Rev Saude Publica & Artigo & 2011 & & \\
\hline VIEIRA, F. S & Rev. Saúde Pública & Artigo & 2012 & & \\
\hline MINISTÉRIO DA SAÚDE & Rev. Saúde Pública & Artigo & 2010 & & \\
\hline CHIEFFI, et al & Rev. Saúde Pública & Artigo & 2010 & & \\
\hline COELHO, et al & Rev. Saúde Pública & Artigo & 2014 & & \\
\hline MACHADO-DOS-SANTOS, & Fiocruz & Artigo & 2011 & \multirow{3}{*}{3} & \multirow{3}{*}{$4,34 \%$} \\
\hline SILVA, & Fiocruz & $\begin{array}{c}\text { Tese } \\
\text { Doutorado } \\
\end{array}$ & 2011 & & \\
\hline OLIVEIRA, & Fiocruz & Artigo & 2007 & & \\
\hline
\end{tabular}




\begin{tabular}{|c|c|c|c|c|c|}
\hline MARQUES, O.R.A. & Rev. Direito Sanit. & Artigo & 2011 & \multirow{3}{*}{3} & \multirow{3}{*}{$4,34 \%$} \\
\hline MARQUES, S.B & Rev. Direito Sanit. & Artigo & 2009 & & \\
\hline TANAKA, & Rev. Direito sanit. & Artigo & 2008 & & \\
\hline BIEHL, et al. & Scielo & Artigo & 2015 & \multirow{3}{*}{3} & \multirow{3}{*}{$4,34 \%$} \\
\hline CATANHEIDE, et al & Scielo & Artigo & 2016 & & \\
\hline MACHADO, et al & Scielo & Artigo & 2013 & & \\
\hline OLIVEIRA, V.E & $\begin{array}{c}\text { Brazilian Political Science } \\
\text { Review }\end{array}$ & Artigo & 2011 & \multirow{2}{*}{2} & \multirow{2}{*}{$2,89 \%$} \\
\hline MENICUCCI, & $\begin{array}{c}\text { Brazilian Political Science } \\
\text { Review }\end{array}$ & Artigo & 2010 & & \\
\hline BORGES, et al & Cad. Saúde Pública & Artigo & 2010 & \multirow{2}{*}{2} & \multirow{2}{*}{$2,89 \%$} \\
\hline CHIEFFI, et al & Cad. Saúde Pública & Artigo & 2009 & & \\
\hline ELIAN, & $\begin{array}{c}\text { Direito Sanitário: Saúde } \\
\text { e Direito }\end{array}$ & Livro & 2010 & \multirow{2}{*}{2} & \multirow{2}{*}{$2,89 \%$} \\
\hline PAULA, P.S & $\begin{array}{c}\text { Direito Sanitário: Saúde } \\
\text { e Direito }\end{array}$ & Livro & 2010 & & \\
\hline SALAZAR, & Ministério da Saúde & Artigo & 2008 & \multirow{2}{*}{2} & \multirow{2}{*}{$2,89 \%$} \\
\hline WANNMACHER, & Ministério da Saúde & Artigo & 2010 & & \\
\hline DALLARI, & Physis & Artigo & 2010 & \multirow{2}{*}{2} & \multirow{2}{*}{$2,89 \%$} \\
\hline FIGUEIREDO, & Physis & Artigo & 2010 & & \\
\hline BONFIM & UERJ & $\begin{array}{c}\text { Tese } \\
\text { Doutorado }\end{array}$ & 2008 & \multirow{2}{*}{2} & \multirow{2}{*}{$2,89 \%$} \\
\hline MACHADO, & UERJ & $\begin{array}{c}\text { Tese } \\
\text { Doutorado }\end{array}$ & 2010 & & \\
\hline COSTA, C.C. & UFF & Artigo & 2008 & \multirow{2}{*}{2} & \multirow{2}{*}{$2,89 \%$} \\
\hline MARTINS, & UFF & Artigo & 2011 & & \\
\hline CONTI, & UNB & $\begin{array}{l}\text { Dissertação } \\
\text { Mestrado }\end{array}$ & 2013 & \multirow{2}{*}{2} & \multirow{2}{*}{$2,89 \%$} \\
\hline ROQUETE, & UNB & $\begin{array}{l}\text { Dissertação } \\
\text { Mestrado }\end{array}$ & 2010 & & \\
\hline BRANDÃO, & Âmbito Jurídico & Artigo & 2015 & 1 & $1,44 \%$ \\
\hline LIVRAMENTO, et al & Amicus Curiae & Artigo & 2011 & 1 & $1,44 \%$ \\
\hline JUNIOR, & Biblioteca Digital FGV & Artigo & 2017 & 1 & $1,44 \%$ \\
\hline BRASIL & $\mathrm{CF}$ & Lei & 1988 & 1 & $1,44 \%$ \\
\hline PEPE, & $\begin{array}{c}\text { Ciência \& Saúde } \\
\text { Coletiva }\end{array}$ & Artigo & 2010 & 1 & $1,44 \%$ \\
\hline BRASIL & Conasems & Artigo & 2011 & 1 & $1,44 \%$ \\
\hline OHLAND, & Revista Direito \& Justiça & Artigo & 2011 & 1 & $1,44 \%$ \\
\hline FERREIRA, & $\begin{array}{l}\text { Faculdade Estácio de } \\
\text { Sá - RJ }\end{array}$ & $\begin{array}{l}\text { Dissertação } \\
\text { Mestrado }\end{array}$ & 2007 & 1 & $1,44 \%$ \\
\hline POMPEI, & Revista Femina & Artigo & 2010 & 1 & $1,44 \%$ \\
\hline
\end{tabular}




\begin{tabular}{|c|c|c|c|c|c|}
\hline REIS JUNIOR, & FGV & $\begin{array}{c}\text { Dissertação } \\
\text { Mestrado }\end{array}$ & 2008 & 1 & $1,44 \%$ \\
\hline BOING, et al & $\begin{array}{c}\text { Revista Health \& Social } \\
\text { Change }\end{array}$ & Artigo & 2010 & 1 & $1,44 \%$ \\
\hline FERRAZ, & $\begin{array}{l}\text { Revista Health and } \\
\text { Human Rights }\end{array}$ & Artigo & 2009 & 1 & $1,44 \%$ \\
\hline SCHEFFER, & Instituto de Saúde & Artigo & 2009 & 1 & $1,44 \%$ \\
\hline ARAÚJO et al & $\begin{array}{l}\text { Curso de Direito } \\
\text { Constitucional }\end{array}$ & Livro & 2005 & 1 & $1,44 \%$ \\
\hline LAFFIN, et al & Medline & Artigo & 2017 & 1 & $1,44 \%$ \\
\hline FARIAS, & PUC Salvador-Ba & $\begin{array}{l}\text { Dissertação } \\
\text { Mestrado }\end{array}$ & 2010 & 1 & $1,44 \%$ \\
\hline COSTA,F.V. & RBPP - Uniceub & Artigo & 2017 & 1 & $1,44 \%$ \\
\hline BRANDAU, et al & Rev Bras Cir Cardiovasc & Artigo & 2005 & 1 & $1,44 \%$ \\
\hline ANDRADE, et al & Rev Med Minas Gerais & Artigo & 2008 & 1 & $1,44 \%$ \\
\hline SANT'ANA, J.M.B & Rev Panam Salud Publica & Artigo & 2009 & 1 & $1,44 \%$ \\
\hline VIEIRA, F. S & Rev. Assoc. Med. Bras & Artigo & 2009 & 1 & $1,44 \%$ \\
\hline FREITAS, & Revista AJURIS & Artigo & 2017 & 1 & $1,44 \%$ \\
\hline MINISTÉRIO DA SAÚDE & Revista BVS & Artigo & 2010 & 1 & $1,44 \%$ \\
\hline ROMERO, & $\begin{array}{l}\text { Revista de Direito } \\
\text { Sanitário }\end{array}$ & Artigo & 2010 & 1 & $1,44 \%$ \\
\hline SANTOS, & Revista DPU & Artigo & 2019 & 1 & $1,44 \%$ \\
\hline COOPER, et al & $\begin{array}{l}\text { The Russel Sage } \\
\text { Foundation }\end{array}$ & Artigo & 2009 & 1 & $1,44 \%$ \\
\hline TORRES, & UFBA & $\begin{array}{c}\text { Dissertação } \\
\text { Mestrado }\end{array}$ & 2013 & 1 & $1,44 \%$ \\
\hline PESSOA, & UFC & $\begin{array}{c}\text { Dissertação } \\
\text { Mestrado }\end{array}$ & 2007 & 1 & $1,44 \%$ \\
\hline PROVIN, & UFG & $\begin{array}{c}\text { Tese } \\
\text { Doutorado }\end{array}$ & 2011 & 1 & $1,44 \%$ \\
\hline VIANNA, & UFMG & Artigo & 2002 & 1 & $1,44 \%$ \\
\hline PAULA, & UFP & Artigo & 2011 & 1 & $1,44 \%$ \\
\hline Anjos & UFRGS & $\begin{array}{c}\text { Dissertação } \\
\text { Mestrado }\end{array}$ & 2009 & 1 & $1,44 \%$ \\
\hline RONSEIN, & UFSC & $\begin{array}{c}\text { Dissertação } \\
\text { Mestrado }\end{array}$ & 2010 & 1 & $1,44 \%$ \\
\hline CUNHA, & Univale & Artigo & 2011 & 1 & $1,44 \%$ \\
\hline WANDERLEY, & Universidade de Marília & $\begin{array}{c}\text { Dissertação } \\
\text { Mestrado }\end{array}$ & 2010 & 1 & $1,44 \%$ \\
\hline MARQUES, S.B & USP & $\begin{array}{c}\text { Tese } \\
\text { Doutorado }\end{array}$ & 2010 & 1 & $1,44 \%$ \\
\hline
\end{tabular}

Fonte: Autora (2019). 


\section{RESULTADOS E DISCUSSÃO}

Os 69 estudos localizados pela busca, compóe a produção científica que aborda o tema dos últimos quatorze anos, sobre o processo de judicialização do acesso a medicamentos de alto custo no Brasil. Há que se considerar que houve uma crescente busca por estudar o tema a partir de 2005 .

O perfil das produções acadêmicas versa sobre as características dos requerentes, o comportamento similar nas decisóes judiciais e, ainda, sobre o percentual dos medicamentos requeridos que apresentam alternativa terapêutica - $(79 \%)$, além das dificuldades apresentadas pelos órgãos públicos para o cumprimento dessas decisões judiciais, conforme é discorrido nos resultados abaixo:

Como mecanismo para identificar as instituiçóes que mais se envolveram com o tema, registrou-se a filiação institucional do primeiro autor informada em cada estudo. Foram informadas 19 instituições de ensino e 23 revistas voltadas para a área acadêmica da saúde, destaque para as revistas de Saúde Pública, Direito Sanitário Scielo e a Fundação Oswaldo Cruz (FIOCRUZ), juntas produziram o maior número de publicaçóes $(\mathrm{n}=17)$, assumindo 24,60\% desse universo, seguida pelo grupo de revistas Brazilian Political Science Review, Cadernos de Saúde Pública, o livro de Direito Sanitário: Saúde e Direito, a instituição Ministério da Saúde, Revista Physis, Universidade Estadual do Rio de Janeiro, Universidade Federal Fluminense, Universidade de Brasília, que juntas publicaram 16 trabalhos $(\mathrm{n}=23,12 \%)$, as demais publicaçóes $(\mathrm{n}=36)$ perfazem um total de $51,84 \%$, correspondem livros, leis, revistas, institutos de ensino e instituiçóes de ensino.

Para os periódicos, merecem destaque a Revista de Saúde Pública, Fiocruz, Revista de Direito Sanitário, Scielo, Brazilian Political Science Review, Cadernos de Saúde Pública, Physis, que juntos são responsáveis pela publicação de 23 trabalhos ( $n=33,27 \%$ ), o restante perfazem um total de $69,18 \%$ da produção.

Por critérios de localização geográfica, considerando estudos apenas de instituições brasileiras, têm-se $54 \%$ composto de estudos em universidades da regiáo sudeste, $25 \%$ na região sul, $18 \%$ na região centro-oeste, e $3 \%$ na região nordeste. Convém ressaltar que não houve nenhum estudo apresentado pela região norte e que ainda vale lembrar que este trabalho é fruto de estudo realizado na regiâo sudeste.

Quanto ao tipo de publicação, em sua maioria apresenta-se como artigo científico, $\mathrm{n}=51 \quad(73,91 \%)$, seguida por dissertaçōes de mestrado, $\mathrm{n}=10 \quad(14,49 \%)$, teses de doutorado $\mathrm{n}=4$ (5,79\%), $\mathrm{n}=3$ livros (4,34\%), lei $\mathrm{n}=1 \quad(1,44 \%)$. Das palavras-chaves utilizadas para inclusão-exclusão da pesquisa, foram destacadas as mais frequentes: judicialização $(n=33)$, medicamento $(n=18)$, decisões judiciais $(n=15)$, sistema único de saúde $(\mathrm{n}=10)$, demandas judiciais $(\mathrm{n}=6)$.

Por se tratar de estudo sobre a judicialização do acesso a medicamentos, esperava-se que essas palavras fossem aparecer referidas em um número maior de resultados, porém, como palavras-chaves, foram mencionadas, respectivamente, em apenas $25 \%$ dos estudos. 
Para Caramelli (2011), Pompei (2011), Brandau et al (2005), Marques (2011); ressaltam a importância das palavras-chaves apropriadas como uma das etapas mais importantes na publicação científica ${ }^{20},{ }^{21},{ }^{22},{ }^{23}$.Quanto à natureza da pesquisa, destacam-se estudos de natureza literalmente empírica $\mathrm{n}=58(67,4 \%)$ em relação àqueles de natureza básica-teórica, $\mathrm{n}=28(32,6 \%) .^{24}$

Em relação aos objetivos da pesquisa sobre a judicialização do acesso a medicamentos de alto custo no Brasil, foram encontrados resultados que cruzados e agrupados por similaridade da área pesquisada e são apresentados de forma esquemática abaixo:

Abordar a construção jurisprudencial da responsabilidade solidária dos entes federativos no que tange ao fornecimento de medicamentos, foi o objetivo principal do estudo de Machado (2013), têm como resultado que o fenômeno da judicialização na saúde pode indicar falhas do sistema público de saúde, uma vez que há solicitaçôes de medicamentos constantes de suas listas. ${ }^{25}$

Reis e Júnior (2008), abordam as principais dificuldades enfrentadas pela Secretaria Municipal de Saúde do Rio de Janeiro para cumprir as determinaçóes emanadas pelo Poder Judiciário em atendimento ao que foi demandado pela sociedade. ${ }^{26}$

Os estudos de Ohland (2011), aborda as características das decisões processuais, discute a responsabilidade solidária dos entes federativos no que tange ao fornecimento de medicamentos, finaliza que o conceito de solidariedade do Código Civil, o Poder Judiciário tem chamado ao processo os três entes federados para figurar no pólo passivo, obrigando-os solidariamente à satisfação da tutela pleiteada. ${ }^{27}$

Borges, et al (2005), discutem a atuação e o comportamento do judiciário nas decisóes, apresenta como resultado que o Poder Judiciário concede os pedidos formulados sem considerar a padronização de medicamentos adotada pelo Ministério da Saúde, exercendo assim uma excessiva intervenção na política de saúde. ${ }^{28}$

Em Romero(2010), Salazar et al (2008), Pepe et al (2010), abordam a jurisprudência do

20 CARAMELLI, B. Resumo: o trailer da comunicação científica, 2011, p.143.

21 POMPEI,L.M. Descritores ou palavras-chave nas bases de dados de artigos científicos, 2011, p.172.

22 BRANDAU,R.;MONTEIRO,R.;BRAILE,D.M. Importância do uso correto dos descritores nos artigos científicos, 2005, p.138.

23 MARQUES,S.B. O direito ao acesso universal a medicamentos no Brasil: diálogos entre o direito, a política e a técnica médica, 2011, p.58.

24 Idem

25 MACHADO, M.A.A, et al. Judicializaçáo do acesso a medicamentos no Estado de Minas Gerais, Brasil, 2013, p.185 - 205.

26 REIS JUNIOR,P.B. A judicializaçáo do acesso a medicamentos na perspectiva da Secretaria Municipal de Saúde do Rio de Janeiro, 2008, p.132 - 142.

27 OHLAND,L. A responsabilidade solidária dos entes da federaçáo no fornecimento de Medicamentos, 2011, p.36 (1).

28 BORGES,D.C.L.;UGÁ,M.A.D. Conflitos e impasses da judicializaçáo na obtençáo de medicamentos: as decisóes de $1^{\mathrm{a}}$ instância nas açóes individuais contra o Estado do Rio de Janeiro, Brasil, 2005, p.122. 
Tribunal de Justiça do Distrito Federal, em relação as ações de medicamentos, resulta que a jurisprudência do TJDF sobre a matéria foi conformada pelo julgamento das açóes por medicamentos antiretrovirais impetradas por pacientes de AIDS contra a SESDF no final da década de 90 e, se consolidou no período estudado, favorecendo a efetivação do acesso ao medicamento, ainda que com impactos relevantes sobre aspectos técnicos, financeiros e de gestão da política de assistência farmacêutica adotada. ${ }^{29,30,31}$

Para Machado et al (2011), analisar o perfil dos requerentes dos medicamentos pleiteados pelas açôes judiciais, ${ }^{32}$ observa-se que dentre os medicamentos não disponíveis no sistema público, $79,0 \%$ apresentavam alternativa terapêutica nos programas de assistência farmacêutica.

As características médico-sanitárias, saúde e direito são discutidas por vários estudos. Pepe et al(2010), discute ainda a adequação das determinaçóes judiciais quanto ao acesso a produtos farmacêuticos, e tem como resultado que a vigilância sanitária tem se tornado locus de produção de saber e ocupado fóruns importantes de produção científica voltados à Saúde Coletiva no Brasil, sendo ela ainda concentrada, assim como outras áreas na Saúde.

Os estudos de Costa et al (2017), buscam investigar o fenômeno da judicialização do direito à saúde, no que atine especificamente aos medicamentos de alto custo. Para isso, analisaram a atuaçáo do Supremo Tribunal Federal e os critérios hermenêuticos utilizados no julgamento dos casos a ele apresentados, averiguando sua pertinência com o princípio da dignidade da pessoa humana. ${ }^{33}$ Oito estudos, com destaque para o de Andrade et al (2008); demonstram a atuação e a organização de um serviço de farmácia de um centro de alta complexidade no tratamento ao câncer visando minimizar os impactos negativos administrativos e orçamentários dos mandados judiciais na gestão da assistência farmacêutica. $^{34}$

Livramento et al (2011), ao invés de analisar a demanda de medicamentos para uma patologia específica, optaram por demandas relativas a um medicamento específico, como é o caso do medicamento Etanercepte, seus pedidos são análogos aos realizados para insulina e, observa-se que mais de $70 \%$ das ações ajuizadas para certos medicamentos são de responsabilidade de um advogado, desta forma, pode sugerir uma relaçấo estreita entre o

\footnotetext{
29 ROMERO,L.C.P. A jurisprudência do Tribunal de Justiça do Distrito Federal em açóes de medicamentos. Revista de Direito Sanitário, 2010, p. 11-59.

30 SALAZAR,A.L; GROU,K.B.; SCHEFFER, M. A judicializaçáo dos anti-retrovirais no Brasil, 2008, p. 82-92.

31 PEPE,V.L.E. et al. A produçáo científica e grupos de pesquisa sobre vigilância sanitária no CNPq, 2010, p. 15(Supl. 3):3341-3350.

32 MACHADO, M.A.A. et al. Judicializaçáo do acesso a medicamentos no Estado de Minas Gerais, Brasil, 2011, p.590-598.

33 COSTA,F.V.;MOTTA,I.D.;ARAÚJO,D.A. Judicializaçáo da saúde: A dignidade da pessoa humana e a atuação do supremo tribunal federal no caso dos medicamentos de alto custo, 2017, p.15-20.

34 ANDRADE,E.I.G.;MACHADO,C.D.;FALEIROS,D.R.SZUSTER,D.A.C.;GUERRA JUNIOR,A.A.; SILVA,G.D.;CHERCHIGLIA, M.L.;ACURCIO,F.A. A judicializaçáo da saúde e a política nacional de assistência farmacêutica no Brasil: gestáo da clínica e medicalizaçáo da justiça, 2008, p.56-85.
} 
advogado e o fabricante do medicamento ${ }^{35}$.

Há ainda autores que estudam o custo da judicialização de medicamentos ofertados pelo Sistema Único de Saúde - SUS na região de Ribeirão Preto-São Paulo, cuja análise demonstra que $16 \%$ dos medicamentos demandados deveriam ser ofertados pelo SUS, é o que retrata o estudo ${ }^{36}$.

Ronsein (2008),Tanaka (2008), aborda a relação entre a prescrição médica e o exercício do direito à saúde é estudada em outra pesquisa ${ }^{37},{ }^{38}$. Por sua vez, há uma concepção, além de outras análises que abordam a judicialização sob a perspectiva do uso racional de medicamentos e do uso de evidências científicas para a indicação terapêutica proposta.

A análise da atual política nacional de medicamentos existente no Brasil, relacionando-a com o processo de judicialização da saúde, no caso deuizamento de açôes individuais destinadas a aquisição de medicamentos ditos excepcionais, é tratada pela pesquisa ${ }^{39}$.

Estudo com similaridade contextual é o apresentado pelo Ministério da Saúde - MS (2009), ao tratar do acesso aos medicamentos de alto custo nas Américas, através de uma cartilha auto-instrucional e ilustrativa, buscando retratar e analisar a atual política nacional de medicamentos existente no Brasil relacionando com o processo de judicialização de medicamentos de alto custo ${ }^{40}$.

Nos estudos de Martins (2011), Menicucci et al (2010), Ministério da Saúde (Brasil) (a) (2010), Ministério da Saúde (Brasil) (b)(2010), Ellian (2010), Lopes (2010), Wanderley (2010), Marques et al (2010), Marques et al (2009), Oliveira et al (2011), Paula (a) (2011), Paula (b) (2010), Reis Junior (2008); têm como interesse principal analisar o impacto ou a interferência das demandas judiciais nas políticas públicas, especialmente na política de medicamentos e na gestão da assistência farmacêutica de medicamentos de

35 LIVRAMENTO,F.A.;SALEH,S.M. O fornecimento gratuito de medicamentos pelo estado: Um estudo do direito fundamental à saúde, pela análise da açấo civil pública que pleiteia análogos de insulina $\mathrm{x}$ açóes individuais, 2011, p.7-15.

36 LAFFIN,N.H.F.; BONACIN,C.A.C. Custos da Saúde: Judicialização de Medicamentos Ofertados pelo SUS, 2012, p.80-85.

37 RONSEIN, J. G. Análise do perfil das solicitaçóes de medicamentos por demanda judicial no Estado de Santa Catarina no período de 2005 a 2008, p. 210-215.

38 TANAKA,O.Y. A judicializaçáo da prescriçáo medicamentosa no SUS ou o desafio de garantir o direito constitucional de acesso à assistência farmacêutica, 2008, p.139-143.

39 FREITAS,D.C. Medicamentos de alto custo no Brasil: Análise da política nacional de medicamentos e balizas para a adoção de critérios de decisóes do poder judiciário, 2019, p.142-156.

40 MINISTÉRIO DA SAÚDE. Secretaria de Vigilância em Saúde. Programa Nacional de DST e Aids. Direitos Humanos e HIV/AIDS: avanços e perspectivas para o enfrentamento da epidemia no Brasil, 2008, p.43-52. 
alto custo no Brasil. ${ }^{41,42,43,44,45,46,47,54,48,49,50,51,52,53}$

Em Dalllari (2010), Cunha (2011), Provin (2011), Ferreira (2007), discutem também a questão sob a perspectiva da Secretaria de Saúde, apresentando dificuldades enfrentadas pelo órgão para efetivação do cumprimento das demandas judiciais, além de apresentam os seus aspectos financeiros. ${ }^{54,55,56,57}$

O trabalho desenvolvido por Biehl (2013), busca estudar o fenômeno por meio da deflagração das açóes judiciais promovidas por portadores de HIV/AIDS a partir da década de 90 e analisadas mais especificamente nos últimos dez anos. As decisóes judiciais obrigavam o executivo a fornecer os medicamentos, garantindo uma sobrevida ao paciente e, em consequência, gerando impacto nas despesas orçamentárias. ${ }^{58}$

$41 \quad$ MARTINS,M.C.R. Possibilidades e limitaçóes da judicializaçáo como instrumento de aperfeiçoamento do exercício do direito à saúde, 2011, p.42-46.

42 MENICUCCI,T.M.G.; MACHADO, J.A. Judicialization of health policy in the definition of access to public goods: Individual rights versus collective rights, 2010, p.20-25.

43 BRASIL.Ministério da Saúde(a). O acesso a medicamentos de alto custo nas américas, 2010, p.62-95.

44 BRASIL.Ministério da Saúde(b). Açóes judiciais: estratégia da indústria farmacêutica para introdução de novos medicamentos,2010, p.421-429.

45 ELIAN,C.O. Efeitos da coisa julgada nas açóes coletivas e o caso da hepatite C.2010, p.429-461.

46 LOPES, L.C.et al. Uso racional de medicamentos antineoplásicos e açóes judiciais no Estado de São Paulo,2010, p.620-628.

47 WANDERLEY,A.W.L, et al. Efetivaçáo do direito fundamental à saúde: fornecimento de medicamentos excepcionais, 2010, p.28-38.

48 MARQUES,O.R.A.;MELO,M.B.;SANTOS,A.P.S. Açóes judiciais no âmbito do sistema único de saúde do brasil, bases legais e implicaçóes: Um estudo de caso em um tribunal da região sudeste, 2010, p.41-66.

49 MARQUES,O.R.A.;MELO,M.B.;SANTOS,A.P.S. O princípio constitucional da integralidade de assistência à saúde e o projeto de lei n. 219/2007: interpretação e aplicabilidade pelo Poder Judiciário,2009, p.64-86.

50 OLIVEIRA,V.E.;NORONHA,L.N.T. Judiciary-executive relations in policy making: The case of drug distribution in the state of São Paulo,2011, p.35-46.

51 PAULA,A.H.R. O direito constitucional sanitário e as açóes judiciais para obtençáo de medicamentos na área oncológica, 2011, p.24-35.

52 PAULA,P.S.;SATURNINO,L.T.M. Descriçáo das demandas do medicamento Etanercepte no Estado de Minas Gerais no período de janeiro a abril de 2008, 2010, p.339-352.

53 REIS JUNIOR,P.B. A judicializaçáo do acesso a medicamentos na perspectiva da Secretaria Municipal de Saúde do Rio de Janeiro, 2008, p.42-56.

54 DALLARI,S.G. - Controle judicial da política de assistência farmacêutica: direito, ciência e técnica, 2010, p. $57-75$.

55 CUNHA,A.E. A judicializaçáo do direito a saúde no Estado de Santa Catarina, 2011, p.168-185.

56 PROVIN,M.P. Demandas judiciais deferidas para medicamentos em Goiânia GO, Brasil, e seus aspectos financeiros, 2011, p. 52-68.

57 FERREIRA,M.G.M. Perfil das demandas judiciais para acesso a Medicamentos no Município de Itaperuna: Uma contribuição sobre novas perspectivas de acesso racional e igualitário, 2007, p.68-85.

58 BIEHL, João;et al. Tratamentos jurídicos: os mercados terapêuticos e a judicializaçáo do direito à saúde, 2013, p.168-185. 
Discute-se ainda ${ }^{59,60}$ a compatibilização entre a lei e as normas técnicas da Assistência, enquanto ${ }^{61}$ estuda as implicações da definição restritiva do princípio da integralidade por uma lei emanada do parlamento.

Ainda possuem aqueles que também estudam e buscam conhecer as características das ações judiciais, por meio do estudo de revisōes sistemáticas de natureza empírica, publicados nos últimos anos para compreender o fenômeno da judicialização no Brasil. ${ }^{62,63}$

Refletir sobre o direito fundamental à saúde e sua aplicação via Sistema Único de Saúde, em pauta o julgamento do Recurso Extraordinário, com repercussão geral, de número $566.471 / \mathrm{RN}$, no qual o relator, Ministro Marco Aurélio de Melo adiantou seu voto ${ }^{64}$. Onze estudos das referências, Vieira et al (2009), Biehl (2013), (2018), Ronsein (2010), Ferraz (2009), Roquete (2010), Torres (2011), Sant'ana et al (2011), Torres (2013), Silva (2011), Coelho et al (2014), Conti (2013), juntos analisam ainda a ocupação dos autores requerentes, os aposentados ou pensionistas são apontados como autores mais frequentes das ações judiciais, em proporções que vão desde 8,9\% a 31,2\% em 16 estudos, é o que aponta. ${ }^{65,66,67,68,69,70,71,72,73,74,75}$

59 SCHEFFER,M. Judicializaçáo e incorporaçáo de tecnologias: o caso dos medicamentos para tratamento da AIDS no Sistema Único de Saúde,2009, p.129-138.

60 ANJOS, R.S. Determinantes e resultados da judicializaçáo da demanda por medicamentos de altocusto no RS: o caso da distribuição do medicamento Interferon em Porto Alegre e Regiáo Metropolitana, 2009.

${ }_{61}$ MACEDO, E.I.; LOPES, L.C.; BARBERATO-FILHO, S. Análise técnica para a tomada de decisão do fornecimento de medicamentos pela via judicial,2011, p.706-713.

62 MACHADO-DOS-SANTOS, S. C. Melhoria da equidade no acesso aos medicamentos no Brasil: os desafios impostos pela dinâmica da competiçâo "extraprę̧o", 2001.

63 FERRAZ, O.L.M. The right to health in the courts of Brazil: Worsening health inequities? Health and Human Rights, 2009, p.33-45.

64 SANTOS, L.F.F.;PINHEIRO,B.O. Acesso universal e gratuito à saúde e o julgamento do RE 566.471/RE, 2018, p.7-22.

65 VIEIRA, F. S.; ZUCCHI, P. Demandas judiciais e assistência terapêutica no Sistema Único de Saúde, 2009, p.672-683.

66 Idem

67 RONSEIN, J. G. Análise do perfil das solicitaçóes de medicamentos por demanda judicial no Estado de Santa Catarina no período de 2005 a 2008, 2010, p.215.

68 Idem

69 ROQUETE, F.L.V. Entre dilemas judiciais e escolhas de políticas: o caso da política pública de assistência farmacêutica, 2010, p.67-85.

70 Idem

71 SANT'ANA, João Mauricio Brambati et al. Racionalidade terapêutica: elementos médico-sanitários nas demandas judiciais de medicamentos,2011, p.714-721.

72 TORRES, I.D.C. Judicialização do Acesso a Medicamentos no Brasil: Uma revisão sistemática, 2013, p. $45-62$

73 SILVA, R.T.B. Conflitos bioéticos decorrentes do acesso a medicamentos órfáos no Brasil: o exemplo da laronidase para o tratamento da mucopolissacaridose tipo I, 2011, p. 56-66.

74 COELHO, T. L. et al. Variáveis jurídicas e de saúde no deferimento de liminares por medicamentos em Minas Gerais, 2014, p.808-816.

75 CONTI, M. A. Avaliaçáo das demandas judiciais por acesso a medicamentos no Distrito Federal, 
Buscar uma melhor compreensão sobre o que é a saúde como preconizada pela Constituição, ponderando sobre o papel do judiciário na persecuçáo desse direito em meio a um cenário de excessiva judicialização, é temática de outro estudo ${ }^{76}$.

Os 82 estudos revisados apresentam achados que ajudam a compreender o fenômeno da judicialização de medicamentos de alto custo no Brasil: as liminares são concedidas na quase totalidade dos casos; parcela considerável das açóes poderia ter sido evitada caso fossem observadas as alternativas terapêuticas do SUS. Os estudos revisados permitem afirmar que os valores gastos com a compra de medicamentos demandados judicialmente comprometam o orçamento do SUS. Enfim, as pesquisas realizadas permitem que se compreenda melhor o fenômeno da judicialização, embora seja recomendável a realização de outros estudos que descrevam características ainda pouco definidas da judicialização do acesso a medicamentos no Brasil.

\section{CONCLUSÃO}

Embora o direito à saúde esteja contemplado na Constituição de 1988, especialmente no artigo 196, carências de natureza gerencial, administrativa e orçamentária entre outras, restringem a capacidade operativa dos órgãos e entidades responsáveis pela sua efetivação desencadeando um fenômeno denominado judicialização do direito à saúde. Este fenômeno é observado quando os indivíduos adotam a iniciativa de recorrer ao Poder Judiciário para garantirem o direito de acesso aos meios e recursos necessários a melhoria de suas condiçôes clínicas. Como o atendimento a saúde deve ser integral, contemplando todas as necessidades do indivíduo, o fenômeno também é observado quando o paciente recorre à justiça para garantir o direito de acesso a medicamentos que deveriam ser fornecidos gratuitamente pelo poder público.

O fenômeno da judicialização na saúde pode indicar falhas do sistema público de saúde, uma vez que há solicitaçóes de medicamentos constantes de suas listas. Todavia, constitui um obstáculo para a prática do uso racional de medicamentos e para a consolidação das premissas da Política Nacional de Medicamentos, principalmente quando são solicitados medicamentos sem comprovação de eficácia e não padronizados pelo Sistema Único de Saúde - SUS.

Outrossim, o desgaste causado pelas negativas, pela desinformação, pela burocracia e pelo tratamento, por vezes, desumanizado acaba por aumentar o sofrimento durante a busca. A judicializaçáo mostrou-se como um dos últimos recursos utilizados pelo cidadão na peregrinação em busca do tratamento medicamentoso em qualquer pesquisa. Percebese que são necessárias açôes públicas no sentido de promover a ampliação do acesso aos medicamentos para a população, através de uma melhor estruturaçáo dos serviços e dispo-

\footnotetext{
2013, p.101-108.

76 COSTA, C.C. A questáo dos medicamentos de alto custo fornecido pelo sus: uma decisão difícil, 2015, p.15-28.
} 
nibilização de recursos para tal, para que os cidadãos possam efetivamente ter um sistema de saúde pautado nos princípios da universalidade e da integralidade.

As pesquisas realizadas permitem que se compreenda melhor o fenômeno da judicialização, embora seja recomendável a realização de outros estudos que descrevam características ainda pouco definidas da judicialização do acesso a medicamentos no Brasil.

O Brasil reconhece o acesso a medicamentos como parte do direito à saúde e adota políticas públicas para propiciar sua garantia. No entanto, a Política Nacional de Assistência Farmacêutica não tem conseguido atender a essa demanda. Em parte, por isso, tem crescido o recurso ao Poder Judiciário para a obtenção desses medicamentos no país.

Os 69 estudos revisados apresentam achados que ajudam a compreender o fenômeno, contextualizar o tema, e ainda auxiliar o leitor a interpretar que uma parcela considerável das açóes poderia ter sido evitada caso fossem observadas as alternativas terapêuticas do SUS. Os estudos revisados não permitem afirmar nem negar que os valores gastos com a compra de medicamentos demandados judicialmente comprometam o orçamento do SUS. Enfim, as pesquisas realizadas permitem que se compreenda melhor o fenômeno da judicialização, embora seja recomendável realizar estudos mais profundos sobre a temática, para que possam descrever características ainda pouco definidas da judicialização do acesso a medicamentos no Brasil.

Nesse prisma, os estudos permitiram vislumbrar ainda que tem ocorrido um aumento exponencial no número de novos processos no decorrer do tempo, além dos gastos para o atendimento destes em todas as esferas da gestão, causando assim desordens no ciclo da assistência farmacêutica, além de prejudicar o financiamento de políticas públicas no Brasil, que se apresenta cada vez mais escasso seu orçamento público.

Tema supereminente, e requer dedicação da comunidade jurídica.

\section{REFERÊNCIAS}

ANDRADE, E. I. G.; MACHADO, C. D.; FALEIROS, D. R..; SZUSTER, D. A. C.; GUERRA JUNIOR, A. A.; SILVA, G. D.; CHERCHIGLIA, M. L.; ACURCIO, F. A. A judicializaçáo da saúde e a política nacional de assistência farmacêutica no Brasil : gestáo da clínica e medicalizaçáo da justiça. Rev Med Minas Gerais, v. 18, n.4, p. S46-S50, 2008.

ANJOS, R.S. Determinantes e resultados da judicializaçáo da demanda por medicamentos de alto-custo no RS: o caso da distribuição do medicamento Interferon em Porto Alegre e Região Metropolitana. 2009. Dissertaçáo (Mestrado). Universidade Federal do Rio Grande do Sul, Porto Alegre, 2009. 
ARAÚJO, L.A.D.;NUNES JÚNIOR,V.S. Curso de Direito Constitucional. 9 ed. São Paulo:Saraiva, 2005.

BIEHL, João; et al. Tratamentos jurídicos: os mercados terapêuticos e a judicializaçáo do direito à saúde. Disponível em: http://www.scielo.br/pdf/hcsm/v23n1/0104-5970-hcsm-23-1-0173.pdf>. Acesso em: 15 Mar 2019.

BOING, A.C.; BLOEMER, N.S.; ROESLER, C. O. Dilema do poder judiciário: Acesso e direito aos medicamentos (the dilemma of the judiciary: Law and acess to medicines). Saúde \& Transformaçáo Social/Health \& Social Change. 2010;2(1):51-57.

BONFIM, R. L. D. Agenda Única de Saúde: a busca do acesso universal e a garantia do direito à saúde. 2008. 164 p. Tese (Doutorado em Saúde Coletiva) - Instituto de Medicina Social, Universidade do Estado do Rio de Janeiro, Rio de Janeiro.

BORGES, D.C.L.; UGÁ, M.A.D. Conflitos e impasses da judicialização na obtenção de medicamentos: as decisōes de $1^{\mathrm{a}}$ instância nas açôes individuais contra o Estado do Rio de Janeiro, Brasil, em 2005. Cad Saude Publica;26(1): 59-69, jan. 2010.

BRANDÁO, L.S. A judicializaçáo da saúde quanto ao fornecimento de medicamentos, 2015. Disponível: http://www.ambitojuridico.com.br/site/?n_link=revista_artigos_ leitura\&artigo_id=15321. Acesso em: 26 Mai 2019.

BRANDAU, R.; MONTEIRO, R.; BRAILE, D.M. Importância do uso correto dos descritores nos artigos científicos. Rev Bras Cir Cardiovasc 2005; 20(1): VII-IX.

BRASIL. Assistência farmacêutica no SUS. Brasília: Conselho Nacional de Secretários de Saúde, 2011. 186p.

.Constituição Federal. Planalto. Portal online. Disponível em: http://www. planalto.gov.br/ccivil_03/constituicao/constituicaocompilado.htm. Acesso em: 13 jan 19.

.Ministério da Saúde. $\mathbf{O}$ acesso a medicamentos de alto custo nas américas.2010. Disponível em: http://bvsms.saude.gov.br/bvs/publicacoes/acesso_medicamentos_alto_custo_americas.pdf. Acesso em: 9 de Fev 2019.

Ações judiciais: estratégia da indústria farmacêutica para introdução de novos medicamentos. Rev. Saúde Pública. São Paulo, v. 44, n. 3, p. 421-429, 2010.

CATANHEIDE; I.D.LISBOA, E.S.SOUZA,L.E.P.F. Característica da Judicializaçáo do acesso a medicamentos no Brasil: Uma revisão sistemática. Disponível em: https:// www.scielosp.org/scielo.php?script $=$ sci_arttext\&pid=S010373312016000401335. Acesso em: 24 Abr 2019.

CHIEFFI, A. L.; BARATA, R. B. Judicialização da política pública de assistência farmacêutica e equidade. Cad. Saúde Pública . Rio de Janeiro, v. 25, n. 8, p. 1839-1849, 2009.

. Ações judiciais: estratégia da indústria farmacêutica para introdução de novos medicamentos. Rev. Saúde Pública. São Paulo, v. 44, n. 3, p. 421-429, 2010. 
COELHO, T. L. et al. Variáveis jurídicas e de saúde no deferimento de liminares por medicamentos em Minas Gerais. Rev. Saúde Pública. São Paulo, v. 48, n. 5, p. 808-816, 2014.

CONTI, M. A. Avaliaçáo das demandas judiciais por acesso a medicamentos no Distrito Federal. 2013. 101 p. Dissertação (Mestrado em Ciências da Saúde) - Faculdade de Ciências da Saúde, Universidade de Brasília, Brasília.

COOPER, H. M.; HEDGES, L. V. The Handbook of research synthesis and meta-analysis. New York: The Russel Sage Foundation, 2009.

COSTA,C.C. A questáo dos medicamentos de alto custo fornecidos pelo SUS: Uma decisão difícil. Disponível em: https://app.uff.br/riuff/bitstream/1/4191/1/TCC.\%20 Clarice\%20Castello\%20Costa.\%20Id\%2011007065..pdf. Acesso em 26 Mai 2019.

COSTA,F.V.; MOTTA, I.D.;ARAÚJO, D.A. Judicializaçáo da saúde: A dignidade da pessoa humana e a atuaçáo do supremo tribunal federal no caso dos medicamentos de alto custo, 2017. Disponível:<https://www.publicacoesacademicas.uniceub.br/RBPP/article/ view/4809. Acesso em: 26 Mai 2019.

CUNHA, A.E. A judicializaçáo do direito a saúde no Estado de Santa Catarina. 2011. Dissertação (Mestrado). Universidade do Vale do Itajaí, Itajaí, 2011.

DALLARI, S.G. - Controle judicial da política de assistência farmacêutica: direito, ciência e técnica. Physis (Rio J.);20(1): 57-75, 2010.

ELIAN, C.O. Efeitos da coisa julgada nas açóes coletivas e o caso da hepatite C. In: Direito Sanitário: Saúde e Direito, um Diálogo Possível / Fernando Aith, Luciana Tarbes Mattana Saturnino, Maria Gabriela Araújo Diniz, Tammy Claret Monteiro (organizadores). - Belo Horizonte : ESP-MG, 2010. p.429-461.

FARIAS, L. C. Mínimo existencial: um parâmetro para o controle judicial para as políticas em saúde. 2010. 149 p. Dissertação (Mestrado em Políticas Sociais e Cidadania) - Universidade Católica do Salvador, Salvador.

FERRAZ, O.L.M. The right to health in the courts of Brazil: Worsening health inequities? Health and Human Rights, v. 11, p. 33-45, 2009.

FERREIRA, M.G.M. Perfil das demandas judiciais para acesso a Medicamentos noMunicípio de Itaperuna: Uma contribuição sobre novas perspectivas de acesso racional e igualitário. 2007. Dissertação (Mestrado). Universidade Estácio de Sá. Riode Janeiro, 2007.

FIGUEIREDO, T.A.; PEPE, V.L.E.; OSORIO-DE-CASTRO, C.G.S. Um enfoque sanitário sobre a demanda judicial de medicamentos. Physis (Rio J.);20(1): 101-118, 2010.

FREITAS, D. C. Medicamentos de alto custo no Brasil: Análise da política nacional de medicamentos e balizas para a adoção de critérios de decisões do poder judiciário. Disponível em: http://www.google.com.br/url?sa=t\&rct=j\&q=\&esrc=s\&source=web\&c$\mathrm{d}=5$ \&ved=2ahUKEwic. Acesso em: 5 Mar 2019. 
JUNIOR, P.B.R. A Judicializaçáo do Acesso a Medicamentos - A Perspectiva da Secretaria Municipal de Saúde do Rio de Janeiro. 2017. Disponível em: https://bibliotecadigital.fgv.br/dspace/bitstream/handle/10438/9058/1423905.pdf. Acesso em: 23 Mai 2019.

LAFFIN, N. H.F.; BONACIN, C. A. C. Custos da Saúde: Judicialização de Medicamentos Ofertados pelo SUS. Disponível em: https://cbc.emnuvens.com.br/articlemedline/viewFile/4312/4312. Acesso em: 20 Mar 2019.

LIVRAMENTO, F.A.; SALEH, S.M. O fornecimento gratuito de medicamentos pelo estado: Um estudo do direito fundamental à saúde, pela análise da ação civil pública que pleiteia análogos de insulina x açóes individuais. Amicus Curiae. 2011;7(7).

LOPES, L.C. et al. Uso racional de medicamentos antineoplásicos e açôes judiciais no Estado de São Paulo. Rev Saude Publica, 44(4): 620-628, ago. 2010.

MACEDO, E.I.; LOPES, L.C.; BARBERATO-FILHO, S. Análise técnica para a tomada de decisão do fornecimento de medicamentos pela via judicial. Rev Saude Publica, 45(4): 706-713, ago. 2011.

MACHADO, F. R. S. A judicializaçáo da saúde no Brasil: cidadanias e assimetrias. 2010. 187 p. Tese (Doutorado em Saúde Coletiva) - Instituto de Medicina Social, Universidade do Estado do Rio de Janeiro, Rio de Janeiro.

MACHADO, Marina Amaral de Ávila; ACURCIO, Francisco de Assis; BRANDÃO, Cristina Mariano Ruas; FALEIROS, Daniel Resende; JUNIOR, Augusto Afonso Guerra; CHERCHIGLIA, Mariângela Leal; ANDRADE, Eli Iola Gurgel. Judicializaçáo do acesso a medicamentos no Estado de Minas Gerais, Brasil. 2013. Disponível em: https://www.scielosp.org/article/rsp/2011.v45n3/590-598/pt/. Acesso em: 10 Mai 201.

MACHADO-DOS-SANTOS, S. C. Melhoria da equidade no acesso aos medicamentos no Brasil: os desafios impostos pela dinâmica da competição "extrapreço", 2011. 180 p. Dissertação (Mestrado em Saúde Pública) - Fundação Oswaldo Cruz, Escola Nacional de Saúde Pública Sérgio Arouca, Rio de Janeiro.

MACHADO, M. A. A. et al. Judicializaçáo do acesso a medicamentos no estado de Minas Gerais, Brasil. Rev. Saúde Pública. São Paulo, v. 45, n. 3, p. 590-598, 2011.

MARQUES, O.R.A.; MELO, M.B.; SANTOS, A.P.S. Açóes judiciais no âmbito do sistema único de saúde do brasil, bases legais e implicaçóes: Um estudo de caso em um tribunal da regiáo sudeste. São Paulo, v.12, n.1, jun. 2011;12(1):41-66.

MARQUES, S.B. O direito ao acesso universal a medicamentos no Brasil: diálogos entre o direito, a política e a técnica médica. 2010. Tese (Doutorado). Universidade de São Paulo, São Paulo, 2010.

- O princípio constitucional da integralidade de assistência à saúde e o projeto de lei n. 219/2007: interpretação e aplicabilidade pelo Poder Judiciário. Rev. direito sanit, 10(2): 64-86, jul.-out. 2009. 
MARTINS, M.C.R. Possibilidades e limitaçóes da judicializaçáo como instrumentode aperfeiçoamento do exercício do direito à saúde. 2011. Dissertação (Mestrado). Universidade Federal Fluminense, Niterói, 2011.

MENICUCCI, T. M. G.; MACHADO, J. A. Judicialization of health policy in the definition of access to public goods: Individual rights versus collective rights. Brazilian Political Science Review. São Paulo, v. 4, n. 1, p. 33-68, 2010.

OHLAND, L. A responsabilidade solidária dos entes da federaçáo no fornecimento de medicamentos. Direito \& Justiça. 2011;36(1).

OLIVEIRA, M. A.; BERMUDEZ, J. A. Z.; OSORIO-DE-CASTRO, C. G. S. Assistência Farmacêutica e acesso a medicamentos. Rio de Janeiro: Fiocruz; 2007. 110p.

OLIVEIRA, V.E.; NORONHA, L.N.T. Judiciary-executive relations in policy making: The case of drug distribution in the state of São Paulo. Brazilian Political Science Review. $2011 ; 5(2)$.

PAULA, A.H.R. O direito constitucional sanitário e as açóes judiciais para obtençáo de medicamentos na área oncológica. 2011. Universidade Federal do Paraná, Curitiba, 2011.

PAULA, P.S.; SATURNINO, L.T.M. Descrição das demandas do medicamento Etanercepte no Estado de Minas Gerais no período de janeiro a abril de 2008. In: Direito Sanitário: Saúde e Direito, um Diálogo Possível / Fernando Aith, Luciana Tarbes Mattana Saturnino, Maria Gabriela Araújo Diniz, Tammy Claret Monteiro (organizadores). - Belo Horizonte : ESP-MG, 2010. p.339-352.

PEPE, V.L.E. et al. A produção científica e grupos de pesquisa sobre vigilância sanitária no CNPq. Ciência \& Saúde Coletiva, 15(Supl. 3):3341-3350, 2010c.

PESSOA, N. T. Perfil das solicitaçóes administrativas e judiciais de medicamentos impetradas contra a secretaria de saúde do estado do Ceará 2007. 168 p. Dissertação (Mestrado em Ciências Farmacêuticas) - Departamento de Farmácia, Universidade Federal do Ceará, Fortaleza.

POMPEI, L.M. Descritores ou palavras-chave nas bases de dados de artigos científicos. Femina; Maio 2010, vol 38, no 5.

PROVIN, M.P. Demandas judiciais deferidas para medicamentos em Goiânia GO, Brasil, e seus aspectos financeiros. 2011. Tese (Doutorado). Universidade Federal de Goiás, Goiânia, 2011

REIS JUNIOR, P.B. A judicializaçáo do acesso a medicamentos na perspectiva da Secretaria Municipal de Saúde do Rio de Janeiro. 2008. Dissertação (Mestrado). Fundação Getúlio Vargas, Rio de Janeiro, 2008.

ROQUETE, F.L.V. Entre dilemas judiciais e escolhas de políticas: o caso da política pública de assistência farmacêutica. 2010. Dissertação (Mestrado). Universidade de Brasília, Brasília, 2010. 
ROMERO, L.C.P. A jurisprudência do Tribunal de Justiça do Distrito Federal em açóes de medicamentos. Revista de Direito Sanitário, v. 11, p. 11-59, 2010.

RONSEIN, J. G. Análise do perfil das solicitaçóes de medicamentos por demanda judicial no Estado de Santa Catarina no período de 2005 a 2008, 2010. 215 p. Dissertaçáo (Mestrado em Farmácia) - Centro de Ciências da Saúde, Universidade Federal de Santa Catarina, Florianópolis.

SALAZAR, A.L; GROU, K.B.; SCHEFFER, M. A judicialização dos anti-retrovirais no Brasil. In: BRASIL. Ministério da Saúde. Secretaria de Vigilância em Saúde. Programa Nacional de DST e Aids. Direitos Humanos e HIV/AIDS: avanços e perspectivas para o enfrentamento da epidemia no Brasil. Brasília : Ministério da Saúde, 2008. p.43-52.

SANT'ANA, J.M.B. et al. Essencialidade e assistência farmacêutica: consideraçóes sobre o acesso a medicamentos mediante ações judiciais no Brasil. Rev Panam Salud Publica. 29(2), 2011b.

.Racionalidade terapêutica: elementos médico-sanitários nas demandas judiciais de medicamentos. Rev. Saúde Pública. São Paulo, v. 45, n.4, p. 714-721, 2011.

SCHEFFER, M. Judicialização e incorporação de tecnologias: o caso dos medicamentos para tratamento da AIDS no Sistema Único de Saúde. In: As açóes judiciais no SUS e a promoçáo do direito à saúde. Keinert, T.M.M; Paula, S.H.B.; Bonfim, J.R.Al. (org.). São Paulo: Instituto de Saúde, 2009. p.129-138.

SILVA, R.T.B. Conflitos bioéticos decorrentes do acesso a medicamentos órfãos no Brasil: o exemplo da laronidase para o tratamento da mucopolissacaridose tipo I. 2011. Tese (Doutorado). Fundação Oswaldo Cruz, Rio de Janeiro, 2011.

SANTOS, L.F.F.;PINHEIRO,B.O. Acesso universal e gratuito à saúde e o julgamento do RE 566.471/RE, 2018, p.7-22. Disponível em: https://revistadadpu.dpu.def.br/index.php/revistadadpu/article/view/58/46. Acesso em: 25 Mai 2019.

TANAKA, O.Y. A judicialização da prescrição medicamentosa no SUS ou o desafio de garantir o direito constitucional de acesso à assistência farmacêutica. Rev. Direito sanit, 9(1): 139-143, 2008.

TORRES, I.D.C. Judicialização do Acesso a Medicamentos no Brasil: Uma revisão sistemática. Disponível em: https://repositorio.ufba.br/ri/bitstream/ri/13137/1/Diss\%20 MP.\%20Izamara\%20Torres\%202013.pdf. Acesso 20 Mai 2019.

VIANNA, L.W. A democracia e os três poderes no Brasil. Belo Horizonte: Editora UFMG, p. 17-42, 2002.

VIEIRA, F. S.; ZUCCHI, P. Demandas judiciais e assistência terapêutica no Sistema Único de Saúde. Rev. Assoc. Med. Bras. São Paulo, v. 55, n. 6, p. 672-683, 2009.

Distorçôes causadas pelas açôes judiciais à política de medicamentos no Brasil. Rev. Saúde Pública. São Paulo, v. 41, n. 2, p. 214-222, 2012. 
WANDERLEY, A.W.L. Efetivaçáo do direito fundamental à saúde: fornecimento de medicamentos excepcionais. 2010. Dissertação (Mestrado). Universidade de Marília, Marília, 2010.

WANNMACHER, L. Importância dos Medicamentos Essenciais em Prescriçáo e Gestáo Racionais. In: BRASIL. Uso Racional de Medicamentos: temas selecionados. Brasília: Ministério da Saúde, 2010, p. 15-20. 